another." It was not our intention to underplay the role of the program, nor to disappoint the thousands of participants. Rather, there was a considerable amount of material to cover and a limited amount of space to do it in. Any history of HTA in the United Kingdom is inevitably a personal reflection, and it is understandable that Professor Gabbay's own account would put the program more center-stage, given his role as former director.

We did acknowledge the central role of the NCCHTA in coordinating HTA efforts in the United Kingdom in recent years and its support for the work of NICE. The number and quality of HTA reports produced by the NHS HTA Programme is indeed impressive and probably surpasses the performance of most, if not all, comparable programs in other jurisdictions. However, the production of reports does not, of itself, guarantee impact. It was our judgment that, in commenting on the past 10 years in the United Kingdom, we should emphasize the role of NICE in using HTAs to issue guidance on the use of health technologies in the NHS. Of course, this is merely our judgment, but one which we believe is consistent with the international view of the recent developments in HTA in the United Kingdom.

Michael Drummond, BS, MCom, DPhil

Professor of Health Economics

Centre for Health Economics

University of York

Heslington, York, North Yorkshire, YO10 5DD

United Kingdom

Email: md18@york.ac.uk

David Banta, MD, MPH

Professor Emiritus

University of Maastricht

Maastricht, The Netherlands

Email: hd.banta@orange.fr

\section{Harmonizing HTA}

doi:10.1017/S0266462310000164

\section{To the Editor:}

Paul Trueman and colleagues (3) have reported on an important issue. Several HTAs on the same topic have been published recently. They have examined four recent HTA reports on drug-eluting stents (DES), demonstrating varying methods and conclusions. All four HTAs included local registry data and economic evaluations in addition to analyses of published research. The authors concluded that the published evidence considered by most of the agencies had "only limited influence on the resulting recommendations."

Although the study by Trueman et al. represents only a small sample of HTA reports - and ought not to lead to broad assumptions as to inconsistency in international HTA-we consider it useful to comment on the methods used in this study, its conclusions, and, in particular, the following statement presented in the discussion section: "this conclusion challenges the EUnetHTA approach."

\section{PROBLEMS IN WORKING METHODS}

The article contains inaccuracies. The HTAs produced by Austrian (LBI) and Belgian (KCE) HTA entities are said to "have no direct link to reimbursement and coverage" $(1 ; 2)$. However, the main KCE mission is to advise policy makers in obtaining an efficient allocation of healthcare resources, and the LBI report had a direct and measurable impact on coverage. Conclusions are sometimes oversimplified. The KCE report, which was incorrectly cited, was interpreted as "advocat[ing] clearly that DES should not be reimbursed," whereas the report actually recommends the consideration of a readjustment of the reimbursement price of DES toward the levels of bare metal stent reimbursement.

Furthermore, the article states that "KCE and LBI considered published evidence on DES but made no attempt to generate primary research.” In fact, a Belgian percutaneous coronary intervention registry was analyzed, and primary research on cost-effectiveness was performed. It was also stated that "these local registry data were used to supplement the published evidence," whereas actually the local data were not applicable and relative risk improvements were based on published meta-analyses.

The summary table of economic evaluations also contains several mistakes such as the omission of countries (Japan and Brazil) and incorrect ranges of outcomes.

\section{APPRAISING A COOKBOOK BY TASTING FOUR MEALS PREPARED WITHOUT USING THE BOOK}

The fundamental problem with the article is that it questions the feasibility of the HTA Core Model approach, even though none of the four HTAs actually used this specific approach.

The article stated that "the core data set was criticized by the HTA bodies and appeared to have had limited influence on the resulting recommendations." As authors of two of the included DES reports, we would like to stress that we did not criticize the idea of a Core Model. Rather, we would see it as a benefit to have a clear structure, accessible guidance, and a common pool of HTA information at hand when preparing local HTAs.

\section{HTA CORE MODEL: WHAT IS IT?}

There were some inaccurate assumptions about the HTA Core Model in the article that probably led to the authors' pessimistic views. 
It was stated that "EUnetHTA approach is expected to provide a Core HTA that can act as a basis for individual country level HTAs with minimal adaptation." This is inaccurate. It is acknowledged within the EUnetHTA Collaboration that the adaptation of Core HTA information for local settings often requires local data collection and analysis.

The Core Model is not a tool that aims to develop normative standards for methods used in HTAs. For example, there are no generally accepted guidelines for economic evaluations and they are unlikely to be developed in the near future, simply because data availability and the purpose of economic evaluations differ in countries. The Core Model does provide methodological guidance, which may, where feasible, translate into voluntary and pragmatic standardization of assessment methods.

The HTA Core Model does not aim to standardize the evidence included in HTAs. The fact that a local HTA often requires primary evidence generation does not diminish the need to identify, analyze, and report all published high quality evidence on the topic. This job can be done collaboratively and could well be the "core work" that the EUnetHTA Collaboration promotes. We believe that this could lead to a more comprehensive evidence base and improved efficiency of HTA across countries, as unnecessary repetition of the same or largely similar work would be reduced.

The HTA Core Model does not aim to obtain harmonized conclusions, and certainly not common pan-European recommendations.

The Core Model is a framework for a standardized structure and reporting of HTAs. The well-framed "question and answer" pairs, called assessment elements, allow the sharing of both work and information. The work of Trueman et al. actually emphasizes this point, and is an argument for improved cooperation between HTA institutions to add value by sharing what can be shared in HTA.

\section{REFERENCES}

1. Kvas E. Drug eluting stents in comparison to uncoated stents in the treatment of cardiopathy. Rapid Assess. LBI-HTA 01, 2006.

2. Neyt M, Van Brabandt H, Devriese S, et al. Drug eluting stents in Belgium: Health technology assessment. Health Technology Assessment (HTA). Bruxelles: Belgian Health Care Knowledge Centre (KCE); 2007. KCE reports 66C.

3. Trueman P, Hurry M, Bending M, Hutton J. The feasibility of harmonizing health technology assessments across jurisdictions: A case study of drug eluting stents. Int J Technol Assess Health Care. 2009;25:455-462.
Iris Pasternack, MD

Email: iris.pasternack@thl.fi

Research Officer

Kristian Lampe, MD

Email: kristian.lampe@thl.fi

Senior Medical Office

Finnish Office for Health Technology Assessment

Finland's National Institute for Health and Welfare

P.O. Box 30

Helsinki, FI-00271

Finland

Chris de Laet, MD, PhD

Email: chris.delaet@kce.fgov.be

Senior Medical Expert

Irina Cleemput, $\mathrm{PhD}$

Email: irina.cleemput@kce.fgov.be

Senior Health Economist

Mattias Neyt, PhD

Email: mattias.neyt@kce.fgov.be

Economic Analysis Expert

Belgian Health Care Knowledge Centre

AC Kruidtuin

Doorbuilding

Kruidtuinlaan 55

Brussels, B-1000

Belgium

Claudia Wild, PD

Email: claudia.wild@hta.lbg.ac.at

Director, Ludwig Boltzmann Institute of

Health Technology Assessment

Garnisongasse 7/20

1190 Vienna

Austria

Finn Børlum Kristensen, MD, PhD

Email: fbk@sst.dk

Adjunct Professor

Faculty of Health Sciences

University of Southern Denmark

Winslфwparken 19

3, Odense C DK 5000

Denmark

Director, European Network for

Health Technology Assessment Secretariat

National Board of Health

67, Islands Brygge

DK 2300 Copenhagen

Denmark 